\title{
废旧镍镉电池真空蒸馏规律的研究 *
}

\author{
朱建新李金惠聂永丰 \\ (清华大学环境科学与工程系, 北京 100084)
}

\begin{abstract}
摘要 在实验室条件下，对镍镉电池的真空蒸馏基本规律进行了探索。分析了温度、压力和时间等工艺因素对 镍镉分离效果的影响, 并对镍镉电池的真空蒸塯机理进行了研究, 为废旧镍镉电池资源化提供了理论依据和实 验数据.
\end{abstract}

关键词：废旧镍镉电池，真空蒸馏，资源化，再生利用技术，环境保护 中图分类号: 0646

我国是镍镉电池的生产和使用大国，每年产生 的废旧镍镉电池大约有 1.2 亿只 ${ }^{[1]}$. 废弃的镍镉电 池中含有大量的重金属镉和镍，对人体十分有害. 早年日本流行的 骨痛病” 就是长期食用 镉米” 和 饮用含镉水引起的 ${ }^{[2]}$.

从资源综合利用的角度考虑, 镍镉电池中含有 大量的有价值金属元素，如能进行再生利用，不但 节约了有限的资源，而且避免了对环境的污染 ${ }^{[3-5]}$. 尤其是我国属于贫镉国家，发展镍镉电池的再生利 用技术就更加重要。

本文用真空蒸馏技术处理镍镉电池，研究了温 度、压力和时间等工艺因素对镍镉分离效果的影 响, 并对镍镉电池的真空蒸馏机理进行了研究, 为 废旧镍镉电池资源化提供了理论依据和实验数据。

\section{1 实验}

\section{1 镍镉电池}

实验中使用的废旧镍镉电池为摩托罗拉公司 提供. 电池型号为 $\mathrm{AA}-\mathrm{NiCd}$, 该种电池主要是构成 电池组作为摩托罗拉对讲机 (Motorola talk about distance) 的电源使用. 其原始组成中含有正负极混 合物 $24.1 \sim 24.5 \mathrm{~g}$, 镀镍铁皮 $4.7 \sim 4.9 \mathrm{~g}$, 正极板 $1.4 \mathrm{~g}$ 和绝缘隔板 $0.4 \mathrm{~g}$.

正负极混合物是实验的研究对象，包括正负极 活性物质、支持镍网及绝缘隔膜纸. 破碎后取样, 利用 ICP-AES（电感耦合等离子体光谱分析）进行 分析,其中 5 种主要金属元素的含量如表 1 所示.

1.2 真空辅助回收系统

经过大量研究镉区域熔炼炉和镉真空蒸馏 炉 ${ }^{[6]}$, 我们自行设计了镍镉电池的真空蒸馏炉, 其 关键技术为使镉的蒸发和凝结在一个石英管中同 时发生。

\section{2 结果与讨论}

表 1 正负极物质中的金属元素含量

Table 1 Typical ICP-AES analyses of active mixture (\%)

\begin{tabular}{cccccc}
\hline \multirow{2}{*}{ No. } & \multicolumn{5}{c}{$m(\%)$} \\
\cline { 2 - 6 } & $\mathrm{Cd}$ & $\mathrm{Co}$ & $\mathrm{Fe}$ & $\mathrm{K}$ & $\mathrm{Ni}$ \\
\hline 1 & 47.42 & 3.61 & 9.11 & 8.23 & 31.63 \\
2 & 46.27 & 3.48 & 9.12 & 8.03 & 33.09 \\
3 & 48.56 & 3.61 & 9.21 & 6.70 & 31.92 \\
\hline
\end{tabular}

2001-11-06 收到初稿, 2001-12-13 收到修改稿. 联系人:朱建新 (E-Mail: zhujianxin00@ mails. tsinghua. edu. cn ). *清华大学环境科学 与工程系重点基金资助项目 


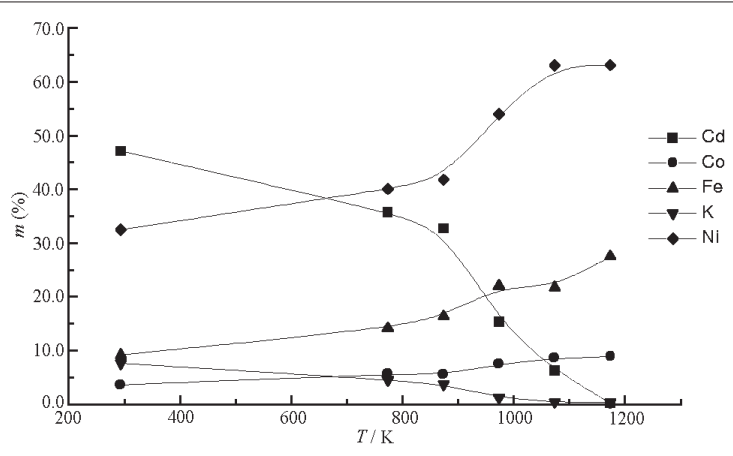

图 1 各金属元素随蒸馏温度的变化规律

Fig. 1 Relationships of contents of metallic elements at different distillation temperatures

\section{1 蒸馏温度的影响}

设定系统压力为 $10 \mathrm{~Pa}$, 分别升温到 773、873、 973、1073 和 $1173 \mathrm{~K}$, 恒温时间 $3 \mathrm{~h}$. 完全冷却后, 取 出蒸馏剩余物进行 ICP-AES, 结果见图 1.

图 1 分别为镍镉电池中五种金属元素 $(\mathrm{Cd} 、 \mathrm{~K}$ 、 $\mathrm{Co} 、 \mathrm{Fe}$ 和 $\mathrm{Ni}$ ) 的含量随蒸馏温度的变化图. 其中 $293 \mathrm{~K}$ 的 $m$ 值为蒸馏前的组成. 从图中可以看出 随着温度的升高, $\mathrm{Cd}$ 和 $\mathrm{K}$ 在反应剩余物中百分含 量呈下降趋势, 而 $\mathrm{Co} 、 \mathrm{Fe}$ 和 $\mathrm{Ni}$ 三种元素的含量呈上 升趋势. 当系统压力为 $10 \mathrm{~Pa}$, 蒸馏温度为 $1073 \mathrm{~K}$, $3 \mathrm{~h}$ 后, 系统可以分离出 $99.3 \%$ 的 $\mathrm{Cd}$; 当温度达到 $1173 \mathrm{~K}$ 时, $\mathrm{Cd}$ 在蒸馏剩余物中的含量几乎为零.

\section{2 反应压力的影响}

分别设定系统压力为 $80 、 40 、 20 、 10 、 0.067 \mathrm{~Pa}$ (实验用真空泵的极限压力), 升温到 $1173 \mathrm{~K}$, 恒温 时间 $3 \mathrm{~h}$. 完全冷却后, 取出蒸馏剩余物进行 ICP-AES, 结果见图 2.

从图 2 中可以看出, 压力的降低有利于 $\mathrm{Cd}$ 的

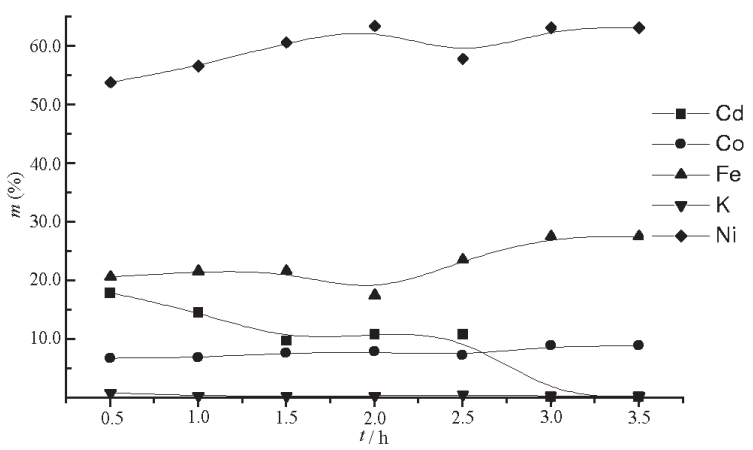

图 3 各金属元素随蒸馏时间的变化规律

Fig. 3 Relationships of contents of metallic elements at different distillation times

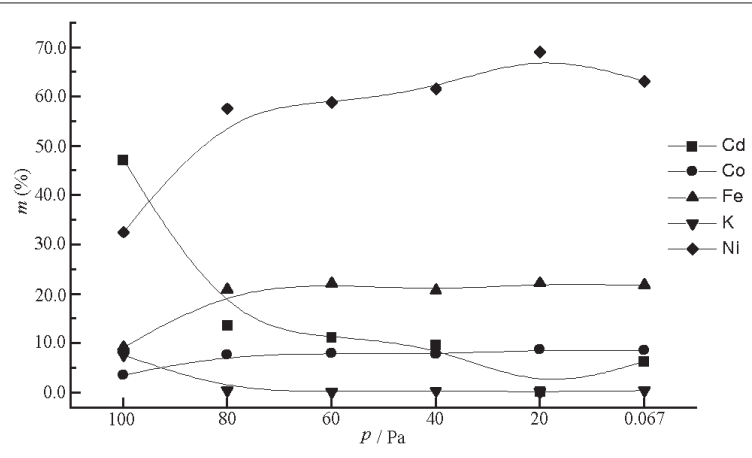

图 2 各金属元素随蒸馏系统压力的变化规律

Fig. 2 Relationships of contents of metallic elements at different distillation pressures

气化分离及 $\mathrm{Co} 、 \mathrm{Fe}$ 和 $\mathrm{Ni}$ 元素的富集. 尤其从 $20 \mathrm{~Pa}$ 到 $10 \mathrm{~Pa}$ 时, $\mathrm{Cd}$ 的分离效果有了显著的提高. 但图 中结果也表明压力并不是越低越好, 太高的真空度 对设备要求高, 而且还可能造成其它金属元素的蒸 发, 达不到镉分离的目的。

\section{3 蒸馏时间的影响}

设定系统压力为 $10 \mathrm{~Pa}$, 温度为 $1173 \mathrm{~K}$, 蒸馏时 间分别为 $0.5 、 1.0 、 1.5 、 2.0 、 2.5 、 3.0$ 和 $3.5 \mathrm{~h}$. 完 全冷却后, 取出蒸馏剩余物进行 ICP-AES 分析, 结 果见图 3.

从图 3 中可以看出, 蒸馏时间是一个非常重要 的影响因素. 随着蒸馏时间的增加, $\mathrm{Cd}$ 在蒸馏剩余 物中的含量迅速降低, 而 $\mathrm{Co} 、 \mathrm{Fe} 、 \mathrm{Ni}$ 的含量迅速升 高. 当温度为 $1173 \mathrm{~K}$, 蒸馏 $3 \mathrm{~h}$ 后, $\mathrm{Cd}$ 的含量几乎 为零.

\section{4 机理初探}

取原始电池的正负极混合物进行 XRD 分析, 其结果如图 4 所示.

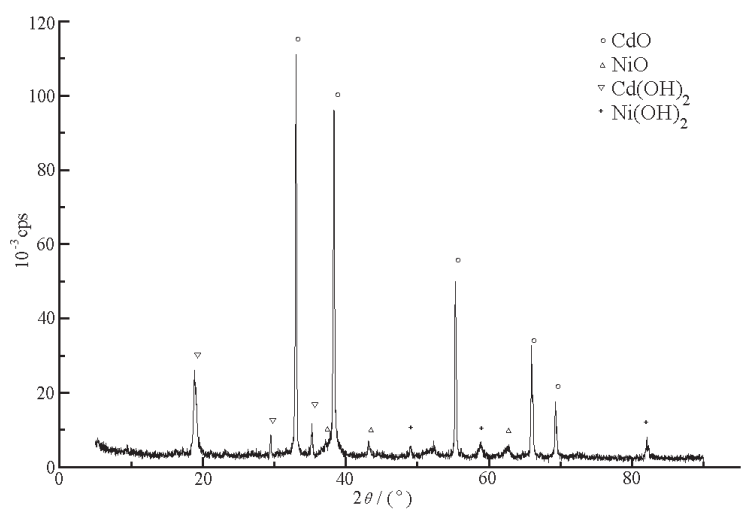

图 4 原始正负极混合物 XRD 图

Fig. 4 Typical XRD analyses of active mixture 
表 2 不同压力下 $\mathrm{CdO}$ 和 $\mathrm{NiO}$ 还原温度

Table 2 The reduction temperature of $\mathrm{CdO}$ and $\mathrm{NiO}$ at different pressures

\begin{tabular}{ccc}
\hline$p / \mathrm{Pa}$ & $T_{\mathrm{CdO}} / \mathrm{K}$ & $T_{\mathrm{Nio}} / \mathrm{K}$ \\
\hline $0.067^{*}$ & 604.96 & 421.16 \\
10 & 673.08 & 491.39 \\
20 & 683.74 & 503.00 \\
40 & 694.75 & 515.18 \\
80 & 706.11 & 527.96 \\
100 & 709.85 & 532.21 \\
101325 & 849.31 & 709.35 \\
\hline
\end{tabular}

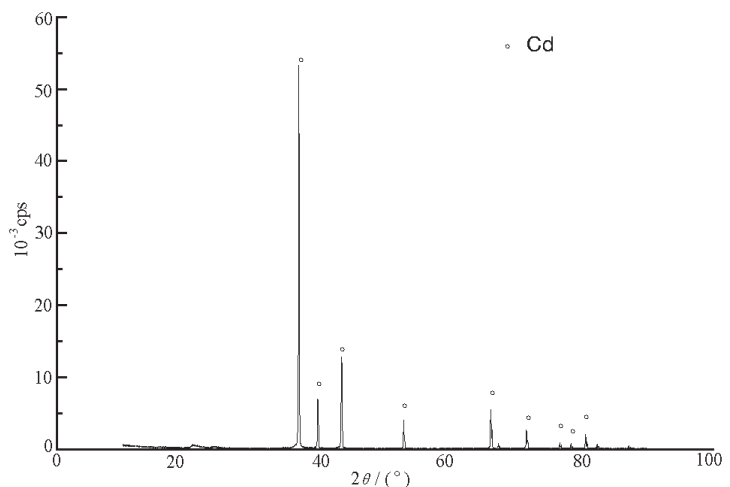

图 5 馏出物的 XRD 图

Fig. 5 XRD spectrum of distillate

表 3 金属镉、镍和铁在不同温度下的蒸气压值

Table 3 The pressure of $\mathrm{Cd}(\mathrm{g}), \mathrm{Ni}(\mathrm{g})$ and $\mathrm{Fe}(\mathrm{g})$ at different temperatures

\begin{tabular}{cccc}
\hline$T / \mathrm{K}$ & $p_{\mathrm{Cd}} / \mathrm{Pa}$ & $p_{\mathrm{Ni}} / \mathrm{Pa}$ & $p_{\mathrm{Fe}} / \mathrm{Pa}$ \\
\hline 773.15 & $1.799 \times 10^{3}$ & $1.982 \times 10^{-16}$ & $1.713 \times 10^{-14}$ \\
873.15 & $1.124 \times 10^{4}$ & $3.230 \times 10^{-13}$ & $1.220 \times 10^{-11}$ \\
973.15 & $4.746 \times 10^{4}$ & $1.124 \times 10^{-10}$ & $2.219 \times 10^{-9}$ \\
1073.15 & $1.514 \times 10^{5}$ & $1.289 \times 10^{-8}$ & $1.512 \times 10^{-7}$ \\
1173.15 & $3.923 \times 10^{5}$ & $6.482 \times 10^{-7}$ & $4.966 \times 10^{-6}$ \\
1273.15 & $8.682 \times 10^{5}$ & $1.738 \times 10^{-5}$ & $9.344 \times 10^{-5}$ \\
\hline
\end{tabular}

由图 4 可以看出, 正负极混合物主要为: $\mathrm{CdO}$ 、 $\mathrm{NiO} 、 \mathrm{Cd}(\mathrm{OH})_{2} 、 \mathrm{Ni}(\mathrm{OH})_{2}$ 、和 $\mathrm{H}_{2} \mathrm{O}$. 因为电池本身含 有导电碳粉, 而且在升温气化过程中, 隔膜纸会发 生分解产生碳氢化合物, 所以系统中有还原剂存 在. 这里将还原剂简化为碳, 系统中发生的主要反 应有

$$
\begin{aligned}
\mathrm{Cd}(\mathrm{OH})_{2} & =\mathrm{CdO}+\mathrm{H}_{2} \mathrm{O} \\
\mathrm{Ni}(\mathrm{OH})_{2} & =\mathrm{NiO}+\mathrm{H}_{2} \mathrm{O} \\
\mathrm{H}_{2} \mathrm{O}(\mathrm{l}) & =\mathrm{H}_{2} \mathrm{O}(\mathrm{g}) \\
\mathrm{CdO}+\mathrm{C} & =\mathrm{Cd}(\mathrm{s})+\mathrm{CO} \\
\mathrm{NiO}+\mathrm{C} & =\mathrm{Ni}(\mathrm{s})+\mathrm{CO} \\
\mathrm{Cd}(\mathrm{s}) & =\mathrm{Cd}(\mathrm{g}) \\
\mathrm{Ni}(\mathrm{s}) & =\mathrm{Ni}(\mathrm{g}) \\
\mathrm{Fe}(\mathrm{s}) & =\mathrm{Fe}(\mathrm{g})
\end{aligned}
$$

为了简化模型, 未将 $\mathrm{K}$ 元素考虑在内. 由计算可 知, 在高温高真空条件下, 式 (1) (3) 可以完全反 应. 对于反应(4)、(5), 受压力影响很大.

表 2 是热力学计算 $\mathrm{CdO}$ 和 $\mathrm{NiO}$ 不同压力下的 还原温度 ${ }^{[7]}$.

从表 2 中可以看出, 当系统压力较低时, $\mathrm{CdO}$ 和 $\mathrm{NiO}$ 的还原温度仅在 $600 \sim 700 \mathrm{~K}$ 之间. 在实验
中, 这个条件能够完全满足. 即该系统中, 镉和镍 是以金属态存在的. 但二者能否实现分离, 还取决 于它们的蒸气压.

表 3 为热力学计算所得金属镉、镍和铁在不同 温度下的蒸气压 ${ }^{[8]}$. 从表中可以看出, 镉在较低的 温度下就具有很高的蒸气压, 而镍和铁即使在 1273 $\mathrm{K}$, 挥发性仍很低. 所以, 真空蒸馏可以达到镍镉分 离的目的,实现纯金属镉回收.

为了证实以上想法, 我们对 $973.15 \mathrm{~K}$ 下的镍镉 电池真空蒸馏馏出物进行了 XRD 分析 (如图 5 所 示). 经分析知, 馏出物确为纯金属镉, 没有镍和铁 存在. ICP-AES 分析证实. 镉纯度高达 $99.85 \%$.

\section{3 结 论}

(1) 压力一定时, 温度的升高能够提高镉的气 化分离效率. 当压力为 $10 \mathrm{~Pa}$, 温度达到 $1173 \mathrm{~K}$ 时, 可以实现镉的完全分离.

(2) 在一定范围内降低压力有利于镉的气化分 离.

(3) 蒸馏时间对镉的分离影响很大. 时间越 长, 分离效果越好. 


\section{References}

1 Hu XR. Battery, 1997, 27(4): 181 [胡行仁. 电池 (Dianchi), 1997, 27 (4): 181]

2 Jing D, Wang TY. Inner Mongolia Environment Protection, 1996，(8)：32 [荆 镝,王同彦.内蒙古环境保护 ( Neimenggu Huanjing Baohu). 1996, (8): 32 ]

3 Yaslunori S, Yoshinori N, Yasuhiko Y. Vacuum, 1999, 53: 101

4 Kasper K P. Industrial Heating, 1996, 34: 241

5 Cox A, Fray D J. Transactions of the Institution of Mining and Metallurgy Section C-Mineral Processing and extractive metallurgy, 1999, 108: C153
6 Robert D P. Unit processes of extractive metallurgy. Beijing: Metallurgy Industry Press, 1982：88 [黄桂柱译. 提取冶金单元 过程．北京: 冶金工业出版社，1982：88]

7 Yang GL. Chinese Nonferrous Metal, 1998, (6): 44 [ 杨桂林. 有色金属 (Youse Jinshu), 1998, (6): 44]

8 Dai YN. Non-ferrous vacuum metallurgy. Beijing: Metallurgy Industry Press，1998：216 [戴永年. 有色金属真空治金. 北京: 冶金工业出版社, 1998: 216]

9 Dai YN, Yang B. Non-ferrous masteries vacuum metallurgy. Beijing: Metallurgy Industry Press, 2000：20 [戴永年, 杨 斌. 有色金属材料真空冶金. 北京：冶金工业出版社, 2000：20]

\title{
Vacuum Thermal Recycling of Used Ni-Cd Batteries *
}

Zhu Jian-Xin Li Jin-Hui Nie Yong-Feng

(Department of Environmental Science and Engineering, Tsinghua University, Beijing 100084)

\begin{abstract}
Recovery of Ni-Cd batteries was studied by a self-designed vacuum-aided recycling system under laboratory conditions. The impacts of temperature, pressure and time were studied respectively. The mechanism of vacuum thermal recycling was also discussed. The results show that, at constant pressure, the increase of temperature can improve the separation efficiency of cadmium. When the temperature is $1173 \mathrm{~K}$, the cadmium can evaporate completely from the residue during $3 \mathrm{~h}$ at $10 \mathrm{~Pa}$. The reduction of pressure in a certain range is effective to separate cadmium by vacuum distillation. Distillation time is a very important factor affecting separation of cadmium.
\end{abstract}

Keywords: Waste Ni-Cd battery, Vacuum distillation, Resource recovery, Recycling technology, Environmental protection

Received: November 6, 2001; Revised: December 13, 2001. Correspondent: Zhu Jian-Xin(E-mail: zhujianxin00@ mails. tsinghua. edu. cn). ${ }^{*}$ The Project Supported by the Department of Environmental Science and Engineering of Tsinghua University 